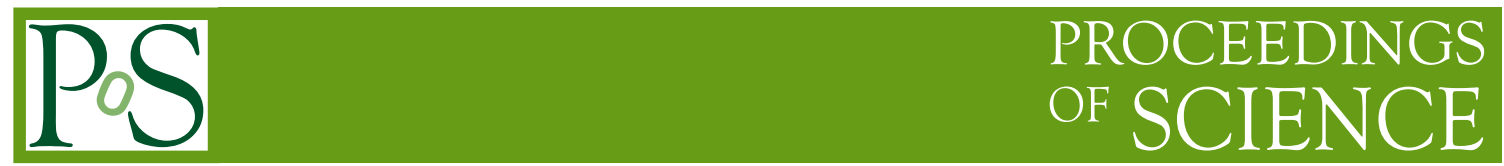

\title{
Quarkonia production in ATLAS and CMS experiments
}

\author{
Stefanos Leontsinis ${ }^{a, *}$ \\ on behalf of the ATLAS and CMS Collaborations \\ ${ }^{a}$ University of Zürich, \\ Winterthurerstrasse 190, Zürich, Switzerland \\ E-mail: Stefanos.Leontsinis@cern.ch
}

Recent results on quarkonia production, using proton-proton collision datasets at $\sqrt{s}=8 \mathrm{TeV}$ and $\sqrt{s}=13 \mathrm{TeV}$ from the ATLAS and CMS experiments at the LHC are presented.

The Eighth Annual Conference on Large Hadron Collider Physics-LHCP2020

25-30 May, 2020

online

${ }^{*}$ Speaker 


\section{Introduction}

Since 2012, the remarkable performance of the LHC [1] together with the efforts of the operation crews of the ATLAS [2] and CMS [3] experiments, managed to store on tape more than $150 \mathrm{fb}^{-1}$ of proton-proton collision data at $\sqrt{s}=8 \mathrm{TeV}$ and $\sqrt{s}=13 \mathrm{TeV}$. Using this dataset, the two experiments updated their measurements with increased precision [4] and kinematic reach [5], and managed to probe rare processes [4, 6-9]. These results on heavy flavour production provide invaluable inputs towards the understanding of QCD.

\section{Measurement of the production cross-section of $\mathrm{J} / \psi$ and $\psi(2 \mathrm{~S})$ mesons at high transverse momentum in pp collisions at $\sqrt{s}=13 \mathrm{TeV}$}

Changing strategy with respect to previous measurements and employing single muon triggers, the ATLAS Collaboration measured $\mathrm{J} / \psi$ and $\psi(2 \mathrm{~S})$ production in the range of high transverse momentum from 60 to $360 \mathrm{GeV}$ and 60 to $140 \mathrm{GeV}$ respectively [5]. This study used $139 \mathrm{fb}^{-1}$ of data collected at $\sqrt{s}=13 \mathrm{TeV}$. The double-differential cross-sections of non-prompt $\mathrm{J} / \psi$ and $\psi(2 \mathrm{~S})$ production is presented in figure 1, where it is also compared with FONLL predictions [10], where a good agreement is observed in the lower $p_{\mathrm{T}}$ region. In the higher $p_{\mathrm{T}}$ region, FONLL predicts higher cross sections.
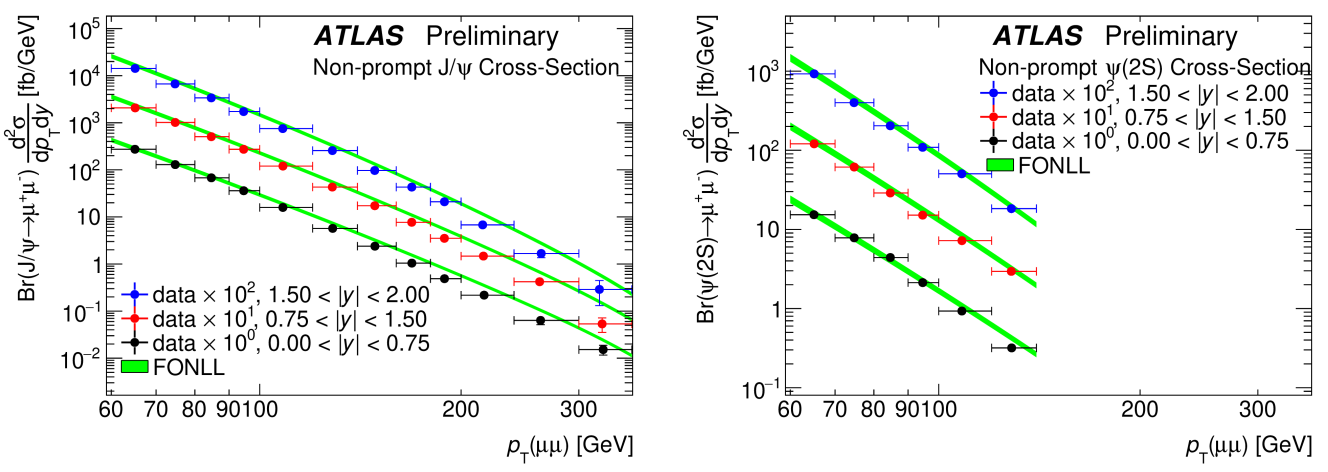

Figure 1: The non-prompt differential cross-section overlaid with FONLL predictions are shown for (left) $\mathrm{J} / \psi$, and (right) $\psi(2 \mathrm{~S})$ mesons, where the horizontal position of each point represents the mean of the weighted $p_{\mathrm{T}}$ distribution for that bin. The data are shown after all relevant corrections are applied, including corrections for acceptance under the assumption of isotropic production. The green shaded bands represent the range of theoretical uncertainty associated to the variations of the scales. Figures from Ref. [5].

\section{Constraints on the $\chi_{\mathrm{c} 1}$ versus $\chi_{\mathrm{c} 2}$ polarizations in proton-proton collisions at $\sqrt{s}=8 \mathrm{TeV}$}

With the measurement of the first two charmonium and the first three bottomonium states being compatible with the unpolarised scenario, the question remained open for the $\chi_{\mathrm{c} 1}$ and $\chi_{\mathrm{c} 2}$ states. In this case, NRQCD predicts an opposite and almost maximal polarisation for the two states. The 
CMS Collaboration measured the polarization of $\chi_{\mathrm{c} 1}$ and $\chi_{\mathrm{c} 2}$ mesons [7] using $19.1 \mathrm{fb}^{-1}$ of pp collisions data at $\sqrt{s}=8 \mathrm{TeV}$. The $\chi_{\mathrm{c}}$ mesons are reconstructed in their $\mathrm{J} / \psi \gamma$ decays, and the study is performed in three $p_{\mathrm{T}}$ bins. The result, as displayed in figure 2 , where the shape parameter $\lambda_{\theta}$ describing the angular momentum composition of the quarkonia is plotted as a function of the particle's $p_{\mathrm{T}} / \mathrm{M}$, is the first significant indication of kinematic difference between the various quarkonia states and is in agreement with the NRQCD prediction.
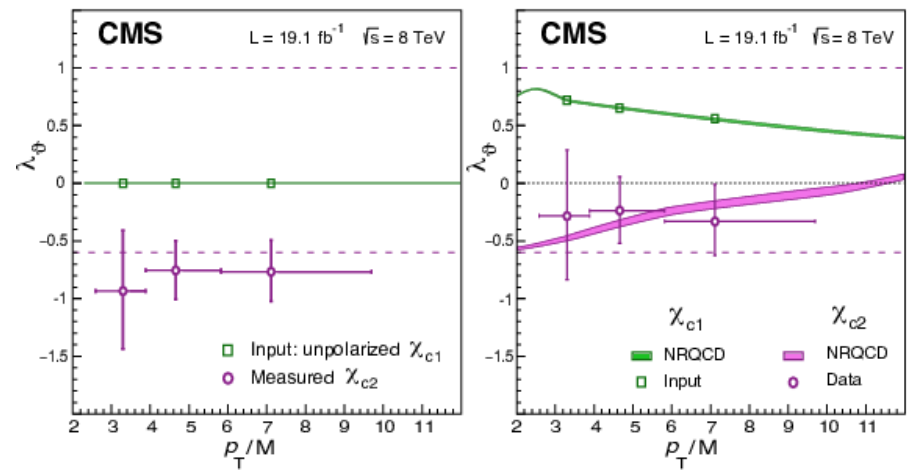

Figure 2: The $\lambda_{\theta}^{\chi_{c 2}}$ values (circles) measured when the $\lambda_{\theta}^{\chi_{c 1}}$ values (squares) are fixed to the unpolarized (left) or the NRQCD (right) scenarios (green curves), as a function of $p_{\mathrm{T}} / M$ of the $\mathrm{J} / \psi$. The purple band on the right is the NRQCD prediction for $\lambda_{\theta}^{\chi_{c 2}}$, while in the unpolarized scenario $\lambda_{\theta}^{\chi_{c 2}}=\lambda_{\theta}^{\chi_{c 1}}=0$. The markers are shown at the average $p_{\mathrm{T}} / M$ values in each bin, the vertical bars represent the total uncertainties, and the horizontal bars the bin widths. The dashed lines indicate the physically allowed range of $\lambda_{\theta}^{\chi c 2}$. Figures from Ref. [7].

\section{Measurement of $\mathrm{J} / \psi$ production in association with a $\mathbf{W}^{ \pm}$boson with pp data at $8 \mathrm{TeV}$}

Studying the associated production of $\mathrm{J} / \psi+\mathrm{W}$ can give valuable input to the Colour Singlet (CS) [11] and Colour Octet (CO) [12] J/ $\psi$ production modelling. The ATLAS Collaboration, extending the studies in the associated production of quarkonia with electroweak bosons [13, 14], measured the ratio of $\mathrm{J} / \psi+\mathrm{W}$ production cross section to the inclusive $\mathrm{W}$ boson production using $20.3 \mathrm{fb}^{-1}$ of proton-proton collisions at $\sqrt{s}=8 \mathrm{TeV}$ [4]. The results are presented first for $\mathrm{J} / \psi$ muons within the ATLAS fiducial volume and then corrected for spin-alignment effects, as shown in figure 3. Using a value of $\sigma_{\text {eff }}=6.3 \mathrm{mb}$ for the double parton scattering and subtracting the DPS component, a comparison is made with a NLO CO model, which is in agreement with the data within uncertainties, but fails to model the high- $p_{\mathrm{T}}$ region, possibly due to the CS contributions missing in the comparison.

\section{Relative cross sections of the $B_{c}^{+}(2 S)$ and $B_{c}^{*+}(2 S)$ states with respect to the $B_{c}^{+}$ state in proton-proton collisions at $\sqrt{s}=13 \mathrm{TeV}$}

The $\mathrm{B}_{\mathrm{c}}^{+}$family of mesons, after the discovery of the ground and its excited states at CDF [15] and the LHC experiments [16, 17], received increased interest towards the understanding of its 

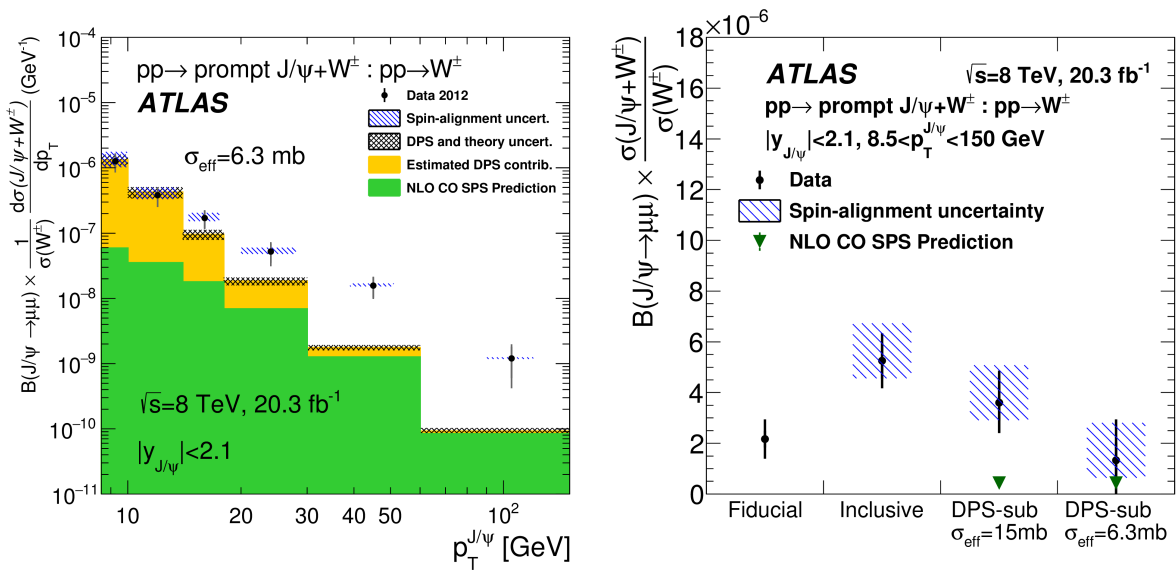

Figure 3: Left: The inclusive differential cross-section ratio measurements and theory predictions. NLO CO SPS predictions are overlaid. The DPS contribution is estimated using $\sigma=6.3 \pm 1.9 \mathrm{mb}$. Right: Production cross-section ratios of $\mathrm{J} / \psi+\mathrm{W}$, relative to inclusive $\mathrm{W}$ production. The first point indicates the cross-section ratio measured in the fiducial volume and the second point shows the ratio corrected for detector acceptance effects on the $\mathrm{J} / \psi$ reconstruction. The third and fourth points illustrate the cross-section ratio after subtraction of the double parton scattering contribution. The DPS-subtracted result is compared with the NLO CO single parton scattering prediction. Figures from Ref. [4].

properties. After the observation of the $\mathrm{B}_{\mathrm{c}}^{+}(2 \mathrm{~S})$ and $\mathrm{B}_{\mathrm{c}}^{*+}(2 \mathrm{~S})$ states [17], the CMS Collaboration using $143 \mathrm{fb}^{-1}$ of pp collisions at $\sqrt{s}=13 \mathrm{TeV}$, measured the cross section ratios of $\mathrm{B}_{\mathrm{c}}^{+}(2 \mathrm{~S})$ to $\mathrm{B}_{\mathrm{c}}^{+}$ $\left(R^{+}\right), \mathrm{B}_{\mathrm{c}}^{*+}(2 \mathrm{~S})$ to $\mathrm{B}_{\mathrm{c}}^{+}\left(R^{*+}\right)$ and $\mathrm{B}_{\mathrm{c}}^{*+}(2 \mathrm{~S})$ to $\mathrm{B}_{\mathrm{c}}^{+}(2 \mathrm{~S})\left(R^{*+} / R^{+}\right)$[8]. No significant dependance on the $p_{\mathrm{T}}$ and $|y|$ of the $\mathrm{B}_{\mathrm{c}}^{+}$mesons is observed for any of these ratios, as shown on figure 4 .
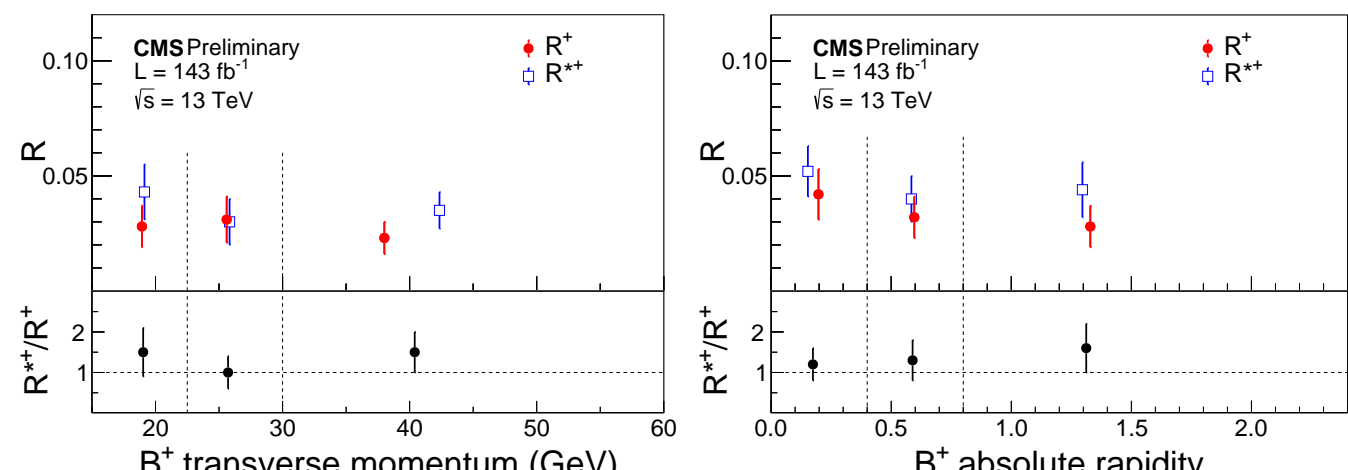

Figure 4: The $R^{+}, R^{*+}$, and $R^{*+} / R^{+}$cross section ratios as a function of the $\mathrm{B}_{\mathrm{c}}^{+} p_{\mathrm{T}}$ (left) and the $\mathrm{B}_{\mathrm{c}}^{+}|y|$ (right). The markers are shown at the average $\mathrm{B}_{\mathrm{c}}^{+} p_{\mathrm{T}}$ or $|y|$ values of the events contributing to each bin. Figures from Ref. [8].

\section{References}

[1] Lyndon Evans and Philip Bryant. LHC Machine. JINST, 3 (2008) S08001. 
[2] ATLAS Collaboration. The ATLAS Experiment at the CERN Large Hadron Collider. JINST, 3 (2008) S08003.

[3] CMS Collaboration. The CMS Experiment at the CERN LHC. JINST, 3 (2008) S08004.

[4] ATLAS Collaboration. Measurement of $J / \psi$ production in association with a $W^{ \pm}$boson with $p p$ data at $8 \mathrm{TeV}$. JHEP, 01 (2020) 095.

[5] ATLAS Collaboration. Measurement of the production cross-section of $J / \psi$ and $\psi(2 S)$ mesons at high transverse momentum in $p p$ collisions at $\sqrt{s}=13 \mathrm{TeV}$ with the ATLAS detector. ATLAS-CONF-2019-047, https://cds.cern.ch/record/2693955/.

[6] CMS Collaboration. Observation of the $\mathrm{Z} \rightarrow \psi \ell^{+} \ell^{-}$decay in pp collisions at $\sqrt{s}=13 \mathrm{TeV}$. Phys. Rev. Lett., 121 (2018) 14.

[7] CMS Collaboration. Constraints on the $\chi_{\mathrm{c} 1}$ versus $\chi_{\mathrm{c} 2}$ polarizations in proton-proton collisions at $\sqrt{s}=8 \mathrm{TeV}$. Phys. Rev. Lett., 124 (2020) 162002.

[8] CMS Collaboration. Relative cross sections of the $\mathrm{B}_{\mathrm{c}}^{+}(2 \mathrm{~S})$ and $\mathrm{B}_{\mathrm{c}}^{*+}(2 \mathrm{~S})$ states with respect to the $\mathrm{B}_{\mathrm{c}}^{+}$state in proton-proton collisions at $\sqrt{s}=13 \mathrm{TeV}$. CMS-PAS-BPH-19-001, https://cds.cern.ch/record/2718820/.

[9] CMS Collaboration. Observation of the $\mathrm{B}_{\mathrm{s}}^{0} \rightarrow \mathrm{X}(3872) \phi$ decay. arXiv:2005.04764.

[10] Matteo Cacciari, Stefano Frixione, Nicolas Houdeau, Michelangelo L. Mangano, Paolo Nason, and Giovanni Ridolfi. Theoretical predictions for charm and bottom production at the LHC. JHEP, 10 (2012) 137.

[11] Bernd A. Kniehl, Caesar P. Palisoc, and Lennart Zwirner. Associated production of heavy quarkonia and electroweak bosons at present and future colliders. Phys. Rev. D, 66 (2002) 114002 .

[12] Gang Li, Mao Song, Ren-You Zhang, and Wen-Gan Ma. QCD corrections to $J / \psi$ production in association with a $W$-boson at the LHC. Phys. Rev. D, 83 (2011) 014001.

[13] ATLAS Collaboration. Measurement of the production cross section of prompt $J / \psi$ mesons in association with a $W^{ \pm}$boson in $p p$ collisions at $\sqrt{s}=7 \mathrm{TeV}$ with the ATLAS detector. JHEP, 04 (2014) 172.

[14] ATLAS Collaboration. Observation and measurements of the production of prompt and nonprompt $J / \psi$ mesons in association with a $Z$ boson in $p p$ collisions at $\sqrt{s}=8 \mathrm{TeV}$ with the ATLAS detector. Eur. Phys. J. C, 75 (2015) 229.

[15] CDF Collaboration. Observation of the $B_{c}$ meson in $p \bar{p}$ collisions at $\sqrt{s}=1.8 \mathrm{TeV}$. Phys. Rev. Lett., 81 (1998) 2432.

[16] ATLAS Collaboration. Observation of an Excited $B_{c}^{ \pm}$Meson State with the ATLAS Detector. Phys. Rev. Lett., 113 (2014) 212004. 
[17] CMS Collaboration. Observation of Two Excited $B_{c}^{+}$States and Measurement of the $B_{c}^{+}(2 S)$ Mass in pp Collisions at $\sqrt{s}=13 \mathrm{TeV}$. Phys. Rev. Lett., 122 (2019) 132001. 